\title{
Visual Servoing from two Special Compounds of Features using a Spherical Projection Model
}

\author{
Romeo Tatsambon Fomena and François Chaumette
}

\begin{abstract}
This paper is concerned with the use of a spherical projection model to design optimal visual features for visual servoing. Here two special targets are considered: a sphere marked with two points on its surface and a sphere marked with a tangent vector to a point on its surface. For these targets we propose a new minimal set of six visual features which can be computed on classical perspective cameras. Using the new set, a classical control method is proved to be globally stable even in the presence of modeling error. In comparison with the previous set originally proposed for the second target, the new set draws a better camera trajectory. Finally, simulation and experimental results confirm the validity of the proposed theoretical results.
\end{abstract}

\section{INTRODUCTION}

In visual servoing, data provided by a vision sensor is used to control the motion of a robot [1]. A vision sensor provides a large spectrum of possible visual features. However, using some of the features could lead to potential problems of stability or visibility if the robot has to achieve a very large displacement [2]. For this reason, we need to design optimal visual features for visual servoing. By optimality, satisfaction of the following criteria is meant: local and as far as possible- global stability of the system, robustness to calibration and to modeling errors, non-singularity, local minima avoidance, satisfactory motion of the system and of the features in the image, and finally maximal decoupling and linear link (the ultimate goal) between the visual features and the degrees of freedom (DOFs) taken into account.

In this context, several methods have been proposed to approach an optimal system behaviour using either 3D data, hybrid (3D and 2D) data or only 2D data. In 3D visual servoing, features in the 3D Cartesian space are used as input to the control scheme [3]. Those features are obtained from the estimation of the relative pose of the target with respect to the camera. This pose can be recovered knowing the geometric model of the target [4]. Pose estimation is thus a key issue in this case. This class of visual servoing is known to provide adequate system motion in the Cartesian space either in the moving frame or in a fixed frame [5]. In this last case, the fact that there is no control of the target in the image might cause visual servoing to fail since visual measurements used for the pose estimation can leave the field of view (FOV). Using the coordinates of several 3D points selected on the target can potentially keep the target in the FOV [6].

R. Tatsambon Fomena is with IRISA / Université de Rennes 1, Campus de Beaulieu, 35 042, Rennes-cedex, France, rtatsamb@irisa.fr

F. Chaumette is with IRISA / INRIA, Campus de Beaulieu, 35 042, Rennes-cedex, France, chaumett@irisa.fr
Another solution to the problem of optimal features modeling is to use hybrid methods that combine 3D and 2D data. This solution has been exploited in $21 / 2 \mathrm{D}$ visual servoing, where a decoupled control scheme with no singularity in the whole task space and tolerating a coarsely calibrated system has been designed [7]. Recently, a similar approach has been applied with a central catadioptric camera [8]. However there are some drawbacks to this method, as the fact that it is more sensitive to image noise (like $3 \mathrm{D}$ visual servoing) than $2 \mathrm{D}$ visual servoing which uses directly features extracted in the image as the control input.

In fact, 2D visual servoing is appealing because of its robustness to camera calibration errors [9], and to image noise. For satisfactory motion of the system in the Cartesian space, the z-axis translational and rotational motions can be decoupled from the other DOFs through a partitioned approach [10]. Another way around the decoupling of the optical axis motions is to use cylindrical coordinates [11]. A generic and intuitive representation of the image of a target can be obtained using 2D moments. Recently, moment invariants theory has been used to determine specific sets of 2D moments whose interaction with the system presents linear and decoupling properties when planar targets are considered [12].

All the above mentioned studies in $2 \mathrm{D}$ visual servoing use a perspective projection model, but other models are also suitable. Considering, as a target, a sphere marked with a tangent vector to a point on its surface (which we refer to as CC target), a spherical projection model has been used to define a global diffeomorphism, which takes into account occlusions and FOV boundary, to control the six DOFs of a system [13]. However, visual features proposed to control the camera orientation are coupled with the camera translational motions [13].

In this paper, the $\mathrm{CC}$ target is revisited and the diffeomorphism is exploited to decouple the orientation control from the camera translational velocities. The obtained decoupling is proved to significantly improve the system behaviour. We also consider a more realistic target composed of a sphere marked with two points on its surface (which we refer to as special sphere). Indeed, from a practical point of view the special sphere is more natural than the $\mathrm{CC}$ target. In addition, the special sphere can be used as an alternative passive target for spacecraft autonomous docking as it has been done with the three spheres in [14]. In the next section the new optimal set of features is presented. Considering the new set of features, a theoretical analysis of the stability and the robustness of a classical control law with respect (w.r.t.) to 


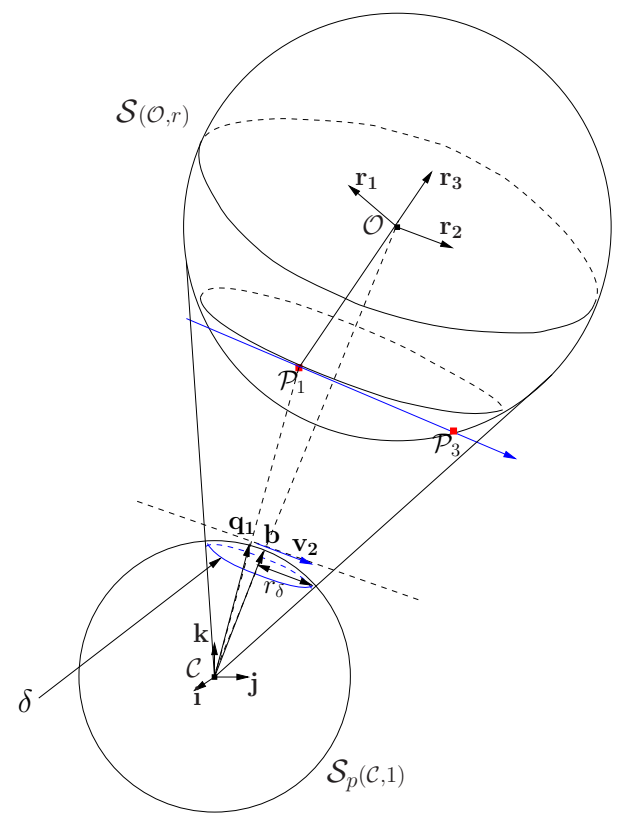

Fig. 1. Spherical projection of the CC target.

modeling error is given in Section III. Finally, simulation and experimental results verifying theoretical results are given in Section IV.

\section{OPTIMAL FEATURES MODELING}

In this section the $\mathrm{CC}$ target is revisited: the orignal set of seven features is first presented and then a new minimal set of six features that improves the system behaviour is designed. Secondly, it is proved that the new minimal set of features can also be used for visual servoing w.r.t. the special sphere. Finally, another new set is selected for both targets and compared with the above mentioned new set.

\section{A. CC target}

We first recall that the interaction matrix $\mathbf{L}_{\mathbf{f}}$ related to a set of features $\mathbf{f} \in \mathbb{R}^{n}$ is defined such that $\dot{\mathbf{f}}=\mathbf{L}_{\mathbf{f}} \mathbf{v}_{c}$ where $\mathbf{v}_{c}=(\boldsymbol{v}, \boldsymbol{\omega}) \in \mathfrak{s e}(\mathbf{3})$ is the instantaneous camera velocity [1]; $\boldsymbol{v}$ and $\boldsymbol{\omega}$ are respectively the translational and the rotational velocities of the camera and $\mathfrak{s e}(\mathbf{3}) \simeq \mathbb{R}^{3} \times \mathbb{R}^{3}$ is the Lie algebra of the Lie group of displacements $\mathbf{S E}(3)$.

Let $\mathcal{S}(\mathcal{O}, r)$ be a sphere of radius $r$ and center $\mathcal{O}$. Let $\mathbf{O}=\left(O_{x}, O_{y}, O_{z}\right)$ be the vector coordinates of $\mathcal{O}$. Let $\mathcal{S}_{p}(\mathcal{C}, 1)$ be the unit sphere of projection center in $\mathcal{C}$. The spherical projection of $\mathcal{S}(\mathcal{O}, r)$ is a dome hat [13]. This dome hat can be caracterised by the circular contour $\delta$ of its base. This contour is pictured in Fig. 1.

As in [13], let us mark the sphere $\mathcal{S}(\mathcal{O}, r)$ with a tangent vector to a feature point $\mathcal{P}_{1}$ on its surface. We obtain the CC target where $\mathcal{O} \mathcal{P}_{1} \perp \mathcal{P}_{1} \mathcal{P}_{3}$ (see Fig. 1). Let ${ }^{c} \mathbf{t}_{o}=\left(t_{x}, t_{y}, t_{z}\right)$ and ${ }^{c} \mathbf{R}_{o}=\left[\begin{array}{lll}\mathbf{r}_{1} & \mathbf{r}_{2} & \mathbf{r}_{3}\end{array}\right]$ be the relative position and orientation respectively of the $\mathrm{CC}$ target w.r.t. to the center of projection frame $\mathcal{F}_{c}=(\mathcal{C}, \mathbf{i}, \mathbf{j}, \mathbf{k})$.

1) Previous approach: Using a spherical projection model, a set of seven visual features for visual servoing w.r.t. the CC target has been proposed in [13]: $\mathbf{s}_{\mathbf{c c}}=\left(r_{\delta}, \mathbf{b}, \boldsymbol{\zeta}\right)$ where

$$
r_{\delta}=\frac{r}{\|\mathbf{O}\|}
$$

is the radius of $\delta$,

$$
\mathbf{b}=\frac{\mathbf{O}}{\|\mathbf{O}\|}=\frac{1}{\|\mathbf{O}\|} c^{c} \mathbf{t}_{o}
$$

is the dome hat summit, and $\zeta$ is equivalent to the $\theta \mathbf{u}$ representation of the rotation matrix $\mathbf{V V}^{*-1}$. Matrix $\mathbf{V}=\left[\begin{array}{lll}\mathbf{v}_{1} & \mathbf{v}_{2} & \mathbf{v}_{3}\end{array}\right]$ is built as follow [13]:

$$
\left\{\begin{array}{l}
\mathbf{v}_{\mathbf{1}}=\mathbf{q}_{\mathbf{1}}=\pi_{s}\left(\mathbf{P}_{1}\right) \\
\mathbf{v}_{\mathbf{2}}=\frac{\Phi_{\mathbf{v}_{\mathbf{1}}} \mathbf{a}_{\mathbf{1}}}{\left\|\Phi_{\mathbf{v}_{\mathbf{1}}} \mathbf{a}_{\mathbf{1}}\right\|}=\frac{\Phi_{\mathbf{v}_{\mathbf{1}}}\left(\pi_{s}\left(\mathbf{P}_{3}\right)-\pi_{s}\left(\mathbf{P}_{1}\right)\right)}{\left\|\Phi_{\mathbf{v}_{\mathbf{1}}}\left(\pi_{s}\left(\mathbf{P}_{3}\right)-\pi_{s}\left(\mathbf{P}_{1}\right)\right)\right\|} \\
\mathbf{v}_{\mathbf{3}}=\mathbf{v}_{\mathbf{1}} \times \mathbf{v}_{\mathbf{2}},
\end{array}\right.
$$

with $\quad \pi_{s}\left(\mathbf{P}_{1}\right)=\mathbf{P}_{i} /\left\|\mathbf{P}_{i}\right\|, \quad i=1 . .3, \quad \mathbf{a}_{13}=\mathbf{P}_{1} \mathbf{P}_{3} \quad$ and $\Phi_{\mathbf{v}_{\mathbf{1}}}=\mathbf{I}_{3}-\mathbf{v}_{\mathbf{1}} \mathbf{v}_{\mathbf{1}}^{\top}$. More precisely, $\mathbf{v}_{\mathbf{2}}$ is a tangent vector to $\mathcal{S}_{p}(\mathcal{C}, 1)$ at the point vector $\mathbf{v}_{\mathbf{1}}$. The interaction matrix corresponding to $\mathbf{s}_{\mathbf{c c}}$ is given by [13]:

$$
\mathbf{L}_{\mathbf{s}_{\mathbf{c c}}}=\left[\begin{array}{cc}
\frac{r_{\delta}^{2}}{r} \mathbf{b}^{\top} & \mathbf{0} \\
-\frac{r_{\delta}}{r}\left(\mathbf{I}_{3}-\mathbf{b} \mathbf{b}^{\top}\right) & {[\mathbf{b}]_{\times}} \\
-\frac{1}{r \beta}\left(\alpha \mathbf{v}_{\mathbf{1}} \mathbf{v}_{\mathbf{3}}^{\top}-\mathbf{v}_{\mathbf{2}} \mathbf{v}_{\mathbf{3}}^{\top}+\mathbf{v}_{\mathbf{3}} \mathbf{v}_{\mathbf{2}}^{\top}\right) & -\mathbf{I}_{3}
\end{array}\right],
$$

where $[\mathbf{b}]_{\times}$is the skew matrix related to $\mathbf{b} ; \alpha$ and $\beta$ are scalar functions of $r_{\delta}, \mathbf{b}, \mathbf{v}_{\mathbf{1}}$ and $\mathbf{v}_{\mathbf{2}}$.

The key point of this original approach is the definition of a diffeormophism that maps a visible subset of the target pose $H=\left({ }^{c} \mathbf{t}_{o},{ }^{c} \mathbf{R}_{o}\right)$ to the image features $\mathbf{s}_{\mathbf{c c}}$. The set $\mathbf{s}_{\mathbf{c c}}$ is not minimal and its interaction matrix $\mathbf{L}_{\mathbf{s}_{\mathrm{cc}}}$ presents a coupling between the orientation control and the camera translational velocities (note the first term in the third row of $\mathbf{L}_{\mathbf{s}_{\mathrm{cc}}}$ ). The following section presents a new set of features that overcomes the two above mentioned problems related to the previous set $\mathbf{s}_{\mathbf{c c}}$.

2) New approach: The previous set $\mathbf{s}_{\mathbf{c c}}$ is not minimal because it uses four parameters $\left(r_{\delta}, \mathbf{b}\right)$ to characterize the image of $\mathcal{S}(\mathcal{O}, r)$ whereas only three parameters are sufficient [1]. That is why the three features

$$
\mathbf{s}_{\mathbf{n}, \mathbf{t}}=\frac{1}{r_{\delta}} \mathbf{b}=\frac{1}{r} c \mathbf{t}_{o}
$$

which are linearly linked to ${ }^{c} \mathbf{t}_{o}$ are preferred [15]. Using a perspective camera it is possible to measure, from the ellipse representing the image of $\mathcal{S}_{(\mathcal{O}, r)}$, the coordinates of $\mathbf{b}$, the radius $r_{\delta}$ and therefore features $\mathbf{s}_{\mathbf{n}, \mathbf{t}}$ [15].

The orientation ${ }^{c} \mathbf{R}_{o}$ of the CC target w.r.t. the camera can be recovered using the diffeomorphism proposed in [13]. From the current and the desired images, the relative orientation ${ }^{c *} \mathbf{R}_{c}$ (of the current frame w.r.t. the desired frame) can be computed. From the matrix ${ }^{c *} \mathbf{R}_{c}$, it is possible to select $\theta \mathbf{u}$ as visual features [7]. We finally obtain the new minimal set of six features $\mathbf{s}_{\mathbf{n}}=\left(\mathbf{s}_{\mathbf{n}, \mathbf{t}}, \theta \mathbf{u}\right)$. The interaction matrix related to $\mathbf{s}_{\mathbf{n}}$ is given by:

$$
\mathbf{L}_{\mathbf{s}_{\mathbf{n}}}=\left[\begin{array}{cc}
-\frac{1}{r} \mathbf{I}_{\mathbf{3}} & {\left[\mathbf{s}_{\mathbf{n}, \mathbf{t}}\right]_{\times}} \\
\mathbf{0} & \mathbf{L}_{\omega}
\end{array}\right]
$$




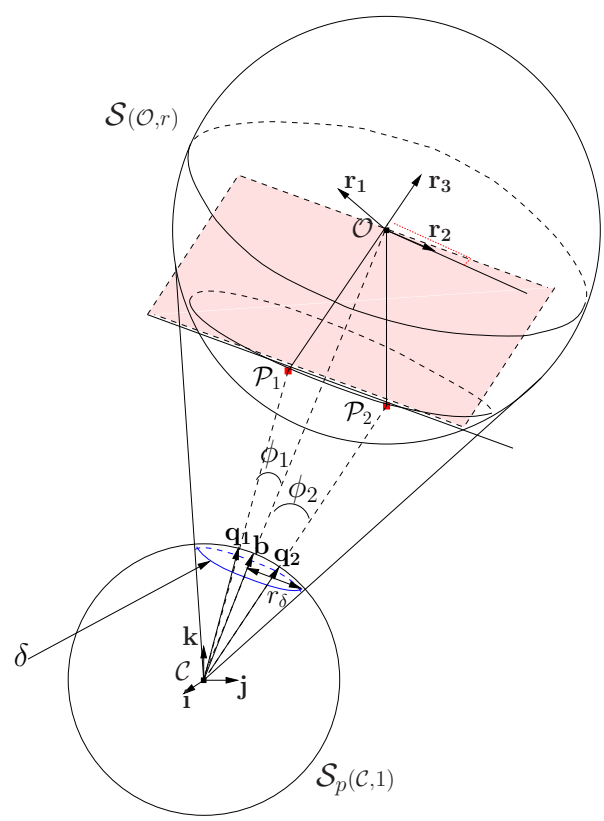

Fig. 2. Spherical projection of a special sphere.

in which $\mathbf{L}_{\omega}$ is given by [7]:

$$
\mathbf{L}_{\omega}=\mathbf{I}_{3}-\frac{\theta}{2}[\mathbf{u}]_{\times}+\left(1-\frac{\operatorname{sinc} \theta}{\operatorname{sinc}^{2} \frac{\theta}{2}}\right)[\mathbf{u}]_{\times}^{2},
$$

where $\operatorname{sinc}(x)=\sin x / x$. The interaction matrix related to $\theta \mathbf{u}$ is completely decoupled from the translational motions, which was not the case when using $\zeta$ (see (4)).

\section{B. Special sphere}

A special sphere is obtained by gluing two feature points $\mathcal{P}_{1}$ and $\mathcal{P}_{2}$ on the surface of a sphere $\mathcal{S}(\mathcal{O}, r)$. Fig. 2 presents the spherical projection of a special sphere. Once again the three features $\mathbf{s}_{\mathbf{n}, \mathbf{t}}$ charaterizing the image of $\mathcal{S}(\mathcal{O}, r)$ can be used to control the camera position.

The control of the camera orientation can also be done by using features $\theta \mathbf{u}$ selected from ${ }^{c *} \mathbf{R}_{c}$. We now show how to determine ${ }^{c} \mathbf{R}_{o}=\left[\begin{array}{lll}\mathbf{r}_{1} & \mathbf{r}_{2} & \mathbf{r}_{3}\end{array}\right]$.

Feature vector $\mathcal{P}_{1} \mathcal{P}_{2}$ is defined such that:

$$
\mathbf{a}_{12}=\mathbf{P}_{1} \mathbf{P}_{2}=\lambda_{1} \mathbf{r}_{3}+\lambda_{2} \mathbf{r}_{2}
$$

where $\left(\lambda_{1}, \lambda_{2}\right) \in \mathbb{R}^{2}$. In [13], as also shown on Fig. 2, the feature point $\mathcal{P}_{1}$ is such that:

$$
\mathbf{r}_{3}=\frac{1}{r}\left({ }^{c} \mathbf{t}_{o}-\mathbf{P}_{1}\right) \text {. }
$$

where $\frac{1}{r} \mathbf{P}_{1}=\frac{\left\|\mathbf{P}_{1}\right\|}{r} \mathbf{q}_{\mathbf{1}}$. It is possible to compute $\left\|\mathbf{P}_{1}\right\| / r$ by applying the cosine rule to the triangle $\left(\mathcal{C}, \mathcal{P}_{1}, \mathcal{O}\right)$ in $\mathcal{F}_{c}$ [13]. We obtain the second degree equation in $\left\|\mathbf{P}_{1}\right\|$

$$
\left\|\mathbf{P}_{1}\right\|^{2}+\|\mathbf{O}\|^{2}-2\left\|\mathbf{P}_{1}\right\|\|\mathbf{O}\| \cos \phi_{1}=r^{2}
$$

where $\phi_{1}=\mathbf{b}^{\top} \mathbf{q}_{\mathbf{1}}$. The two solutions depending on $\sigma= \pm 1$ are given by

$$
\left\|\mathbf{P}_{1}\right\|=\frac{r}{r_{\delta}}\left(\cos \phi_{1}+\sigma \sqrt{r_{\delta}^{2}-\sin ^{2} \phi_{1}}\right)
$$

from which it is easy to deduce $\mathbf{P}_{1} / r=\left(\left\|\mathbf{P}_{1}\right\| / r\right) \mathbf{q}_{\mathbf{1}}$. The choice of $\sigma=-1$ is related to the visibility condition defined in [13]. At this point we can conclude that $\mathbf{r}_{3}$ can be computed from the image of the target. Likewise $\mathbf{P}_{1} / r$, we obtain

$$
\mathbf{P}_{2} / r=\left(\left(\cos \phi_{2}-\sqrt{r_{\delta}^{2}-\sin ^{2} \phi_{2}}\right) / r_{\delta}\right) \mathbf{q}_{\mathbf{2}}
$$

where $\phi_{2}=\mathbf{b}^{\top} \mathbf{q}_{\mathbf{2}}$ with $\mathbf{q}_{\mathbf{2}}=\mathbf{P}_{2} /\left\|\mathbf{P}_{2}\right\|$. From (11) and (12), it is therefore possible to compute

$$
\frac{1}{r} \mathbf{a}_{12}=\frac{1}{r}\left(\mathbf{P}_{2}-\mathbf{P}_{1}\right)
$$

From (13) and (8) we obtain:

$$
\frac{\lambda_{2}}{r} \mathbf{r}_{\mathbf{2}}=\left(\frac{1}{r} \mathbf{a}_{\mathbf{1 2}}-\left(\left(\frac{1}{r} \mathbf{a}_{12}\right)^{\top} \mathbf{r}_{\mathbf{3}}\right) \mathbf{r}_{\mathbf{3}}\right),
$$

from which we easily deduce $\mathbf{r}_{2}$. Finally we have $\mathbf{r}_{1}=\mathbf{r}_{2} \times \mathbf{r}_{3}$ which gives us ${ }^{c} \mathbf{R}_{o}$.

To conclude, it is possible to compute the partial pose of the special sphere and therefore to design the set of six features $\mathbf{s}_{\mathbf{n}}=\left(\mathbf{s}_{\mathbf{n}, \mathbf{t}}, \theta \mathbf{u}\right)$ for visual servoing w.r.t. special spheres.

\section{Discussion}

From the new set $\mathbf{s}_{\mathbf{n}}=\left(\mathbf{s}_{\mathbf{n}, \mathbf{t}}, \theta \mathbf{u}\right)$, it is possible to design another new set $\mathbf{s}=\left(\mathbf{s}_{\mathbf{t}}, \theta \mathbf{u}\right)$ which fully decouples the control of the translation from the camera rotational motions. Indeed, if we use

$$
\mathbf{s}_{\mathbf{t}}=\left(\mathbf{s}_{\mathbf{n}, \mathbf{t}}^{*}-{ }^{c *} \mathbf{R}_{c} \mathbf{s}_{\mathbf{n}, \mathbf{t}}\right),
$$

we obtain:

$$
\mathbf{L}_{\mathbf{s}}=\left[\begin{array}{cc}
\frac{1}{r}{ }^{c *} \mathbf{R}_{c} & \mathbf{0} \\
\mathbf{0} & \mathbf{L}_{\omega}
\end{array}\right] .
$$

Let ${ }^{\mathbf{c} *} \mathbf{t}_{\mathbf{c}}$ be the relative position of the current camera frame w.r.t. the desired camera frame. We can note that $\mathbf{S}_{\mathbf{t}}=\frac{1}{r}{ }^{\mathbf{c} *} \mathbf{t}_{\mathbf{c}}$ corresponds to a classical 3D visual servoing method where there is no control of the target in the image. In addition, there is no more a linear link between the visual features $s_{t}$ and the translational velocities $\boldsymbol{v}$. We therefore prefer the set $\mathbf{s}_{\mathbf{n}}=\left(\mathbf{s}_{\mathbf{n}, \mathbf{t}}, \theta \mathbf{u}\right)$ which is nearly-linear linked to the camera velocities $\mathbf{v}_{c}$ (see (6)) while enabling control of the target in the image. Finally it is important to note that when either $\mathbf{P}_{1}, \mathbf{P}_{2}$ or $\mathbf{P}_{3}$ is occluded or out of the camera FOV, feature $\theta \mathbf{u}$ can not be computed.

\section{STABILITY ANALYSIS TO MODELING ERROR}

In this section, using the set $\mathbf{s}_{\mathbf{n}}$, a theoretical proof of the stability of a classical control law to modeling error is given. We use the classical control law

$$
\mathbf{v}_{c}=-\lambda{\widehat{\mathbf{L}_{\mathbf{n}}}}^{-1}\left(\mathbf{s}_{\mathbf{n}}-\mathbf{s}_{\mathbf{n}}^{*}\right)
$$

where $\mathbf{v}_{c}$ is the camera velocity sent to the low level robot controller, $\lambda$ is a positive gain and ${\widehat{\mathbf{L}_{\mathbf{n}}}}^{-1}$ is the inverse of an approximation of the interaction matrix related to $\mathbf{s}_{\mathbf{n}}$. We suppose that all special marks of each target are visible and we assume that $\mathcal{O} \mathcal{P}_{1} \perp \mathcal{P}_{1} \mathcal{P}_{3}$ for the $\mathrm{CC}$ target. In addition, 
we suppose a perfectly calibrated system and the absence of image processing errors. The only $3 \mathrm{D}$ parameter in $\mathbf{L}_{\mathbf{s}_{\mathbf{n}}}$ is the radius $r$ of $\mathcal{S}(\mathcal{O}, r)$. Incorporating an error on the estimation $\widehat{r}$ of $r$ leads to the closed-loop system equation:

$$
\dot{\mathbf{s}_{\mathbf{n}}}=-\lambda \mathbf{L}_{\mathbf{s}_{\mathbf{n}}}{\widehat{\mathbf{L}_{\mathbf{s}_{\mathbf{n}}}}}^{-1}\left(\mathbf{s}_{\mathbf{n}}-\mathbf{s}_{\mathbf{n}}^{*}\right)
$$

with

$$
\widehat{\mathbf{L}_{\mathbf{s}_{\mathbf{n}}}}=\left[\begin{array}{cc}
-\frac{1}{\widehat{r}} \mathbf{I}_{3} & {\left[\mathbf{s}_{\mathbf{n}, \mathbf{t}}\right]_{\times}} \\
\mathbf{0} & \mathbf{L}_{\omega}
\end{array}\right] .
$$

The global asymptotic stability is obtained if $\mathbf{L}_{\mathbf{s}_{\mathbf{n}}}{\widehat{\mathbf{L}_{\mathbf{s}_{\mathbf{n}}}}}^{-1}>0$. We have:

$$
\mathbf{L}_{\mathbf{s}_{\mathbf{n}}}{\widehat{\mathbf{L}_{\mathbf{n}}}}^{-1}=\left[\begin{array}{cc}
\frac{\widehat{r}}{r} \mathbf{I}_{3} & \left(-\frac{\widehat{r}}{r}\left[\mathbf{s}_{\mathbf{n}, \mathbf{t}}\right]_{\times}+\left[\mathbf{s}_{\mathbf{n}, \mathbf{t}}\right]_{\times}\right) \mathbf{L}_{\omega}^{-1} \\
\mathbf{0} & \mathbf{I}_{3}
\end{array}\right] .
$$

Since $\mathbf{L}_{\mathbf{S}_{\mathbf{n}}}{\widehat{\mathbf{L}_{\mathbf{n}}}}^{-1}$ is a square matrix, its determinant can be calculated:

$$
\operatorname{det}\left(\mathbf{L}_{\mathbf{s}_{\mathbf{n}}}{\widehat{\mathbf{L}_{\mathbf{s}_{\mathbf{n}}}}}^{-1}\right)=\left(\frac{\widehat{r}}{r}\right)^{3}
$$

We have thus

$$
\mathbf{L}_{\mathbf{s}_{\mathbf{n}}}{\widehat{\mathbf{L}_{\mathbf{s}_{\mathbf{n}}}}}^{-1}>0 \Longleftrightarrow \widehat{r}>0 .
$$

This condition is also necessary since if $\widehat{r} \leq 0$ then $\mathbf{L}_{\mathbf{s}_{\mathbf{n}}}{\widehat{\mathbf{L}_{\mathbf{s}_{\mathbf{n}}}}}^{-1} \leq 0$ and the system diverges.

The robutness domain w.r.t. modeling error is thus very large: $\widehat{r} \in] 0,+\infty[$. In practice, it means that with a rough value of the radius of either the CC target or the special sphere, the system still converges.

\section{RESULTS}

In this section, we validate the choice of visual features using a simulator and a six DOFs robotic system equipped with a perspective camera.

\section{A. Simulation results}

1) Special sphere: Here we validate the set of features $\mathbf{s}_{\mathbf{n}}$ proposed for a special sphere. In the target frame, special marks $\mathcal{P}_{1}$ and $\mathcal{P}_{2}$ are sticked to $\mathcal{S}(\mathcal{O}, r)$ such that: $\mathbf{O P}_{1}=r(0,1 / 2,-\sqrt{3} / 2) \quad$ and $\quad \mathbf{O P}_{2}=r(1 / 2,0,-\sqrt{3} / 2)$. Relative to the desired pose, the initial camera pose consists of translation and orientation displacements. Fig. 3 shows features error trajectories and camera velocities. The obtained results confirm the validity of the set of features $\mathbf{s}_{\mathbf{n}}$ for visual servoing w.r.t. special spheres.

2) Comparison of different sets: We now use CC target and compare the three sets of visual features listed in Table I. At the beginning, the relative pose of the camera in the desired frame is made up of translational and rotational motions. Fig. 4 shows the different camera and image feature trajectories. As expected, the use of features $\mathbf{s}$ shows a straight line trajectory of the camera in the Cartesian space. In addition, $\mathbf{s}_{\mathbf{n}}$ plots a shorter camera trajectory compared to $\mathbf{s}_{\mathbf{c c}}$ and straight line trajectories in the image for $\mathbf{b}, \mathbf{P}_{1}$ and $\mathbf{P}_{3}$, while it is not the case for $\mathbf{s}_{\mathbf{c c}}$. Finally, we can note that the image trajectory of feature point $\mathbf{b}$ can potentially get out of the image when using $\mathbf{s}$. That is why the use of the set $\mathbf{s}$ is not recommended and $\mathbf{s}_{\mathbf{n}}$ is preferred.

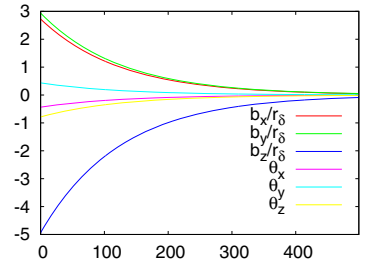

(a)

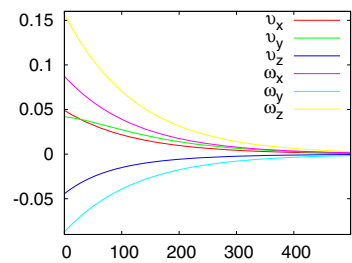

(b)
Fig. 3. Special sphere. (a) $\mathbf{s}_{\mathbf{n}}$ error. (b) Camera velocities (m/s and deg/s).

TABLE I

THREE SETS OF FEATURES TO COMPARE

\begin{tabular}{|c|}
\hline Previous set [13]: $\mathbf{s}_{\mathbf{c c}}=\left(r_{\delta}, \mathbf{b}, \zeta\right)$ \\
\hline New chosen set: $\mathbf{s}_{\mathbf{n}}=\left(\frac{1}{r_{\delta}} \mathbf{b}, \theta \mathbf{u}\right)$ \\
\hline New set: $\mathbf{s}=\left(\frac{1}{r} \mathbf{c}^{*} \mathbf{t}_{\mathbf{c}}, \theta \mathbf{u}\right)$ \\
\hline
\end{tabular}

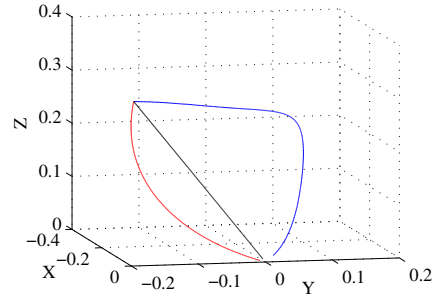

(a)

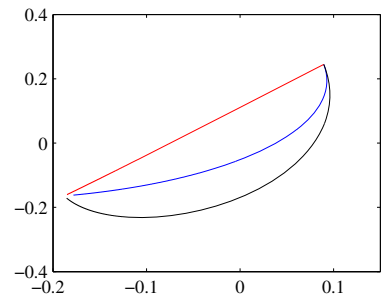

(c)

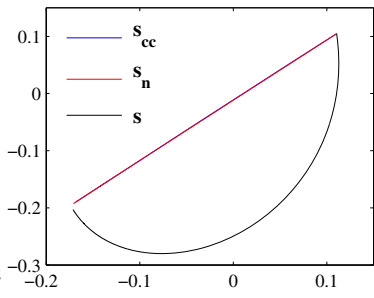

(b)

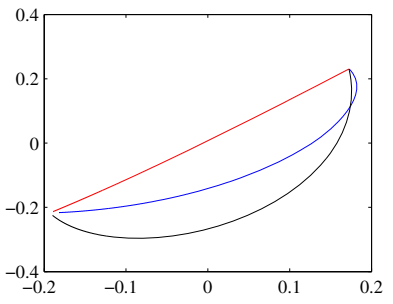

(d)
Fig. 4. System behaviour in simulation. (a) Camera trajectory (m) expressed in the desired frame. (b) Feature point $\mathbf{b}$ trajectory (m) in the image. (c) Special mark $\mathbf{P}_{1}$ trajectory (m) in the image. (d) Special mark $\mathbf{P}_{3}$ trajectory (m) in the image.

\section{B. Experimental results}

Using CC target, we first compare the behaviour of a six DOFs robotic system using $\mathbf{s}_{\mathbf{c c}}$ and $\mathbf{s}_{\mathbf{n}}$. Then, considering both sets, we validate the stability to modeling error and verify the stability to calibration errors. Finally, using a nonspherical decoration balloon and the new set of features $\mathbf{s}_{\mathbf{n}}$, we validate the robustness of the control law w.r.t. strong modeling error.

1) CC target: Let us mention that, in this part, all the experiments have been first tested with $\mathbf{s}_{\mathbf{c c}}$. The reason is that there exist some camera displacements between the desired and the initial poses such that the system is driven to a joint 


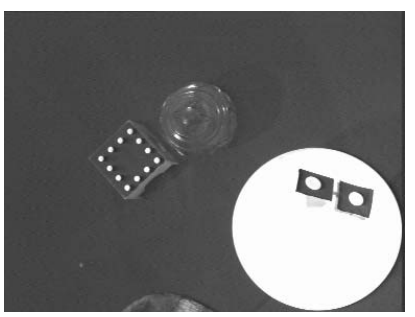

(a)

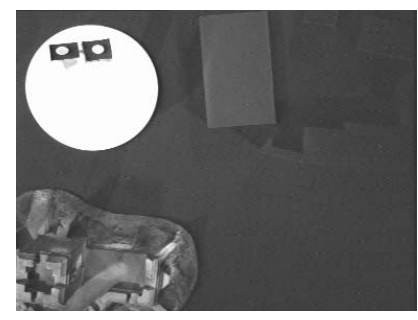

(b)
Fig. 5. CC target. (a) Desired image. (b) Initial image.

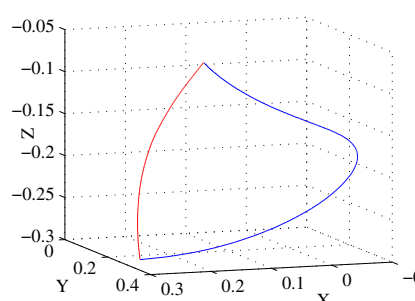

(a)

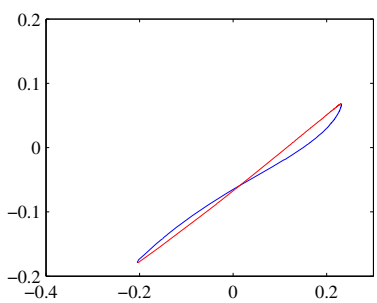

(c)

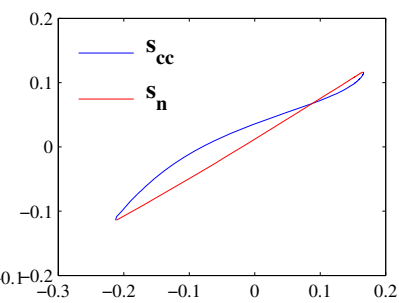

(b)

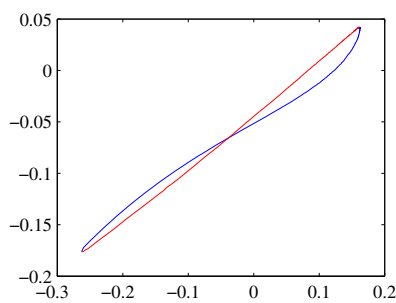

(d)
Fig. 6. Six DOFs robotic system behaviour. (a) Camera trajectory (m) in the cartesian space. (b) Feature point $\mathbf{b}$ trajectory $(\mathrm{m})$ in the image. (c) Special mark $\mathbf{P}_{1}$ trajectory (m) in the image. (d) Special mark $\mathbf{P}_{3}$ trajectory (m) in the image.

limit with $\mathbf{s}_{\mathbf{c c}}$ whereas it converges using $\mathbf{s}_{\mathbf{n}}$. This can be explained by the coupling between the rotational and the translational motions of the camera.

The target is a $9.5 \mathrm{~cm}$ white special spherical ball. Fig. 5 pictures the initial and desired images used for each experiment. The same gain $\lambda=0.1$ has been used in the ideal case, while in the modeling and calibration errors cases, an adaptive gain has been used.

a) Ideal case: We consider the exact value of the radius of the sphere and a correct camera calibration values. The camera and feature trajectories are given in Fig. 6. We can see that, as expected either in the image plane or in the Cartesian space, the trajectories using $\mathbf{s}_{\mathbf{n}}$ are shorter than the ones using $\mathbf{s}_{\mathbf{c c}}$. In addition, and as expected also, the new set $\mathbf{s}_{\mathbf{n}}$ plots an exponential decrease of the camera rotational velocities with less overshoots in the translational velocities (compare Fig. 7(c) and Fig. 7(d)). The first part of the attached video illustrates the differences between the previous and the new sets.

b) Modeling error: We consider the case where $\widehat{r}=0.5 r$. The system converges for the previous and the new sets while the stability to modeling error has been formally proved (in this paper) only for the new set $\mathbf{s}_{\mathbf{n}}$.

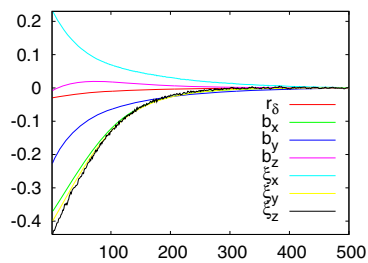

(a)

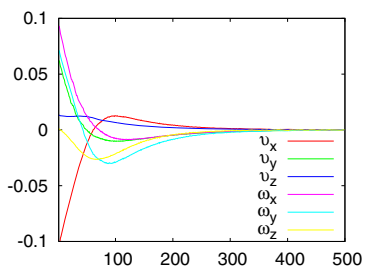

(c)

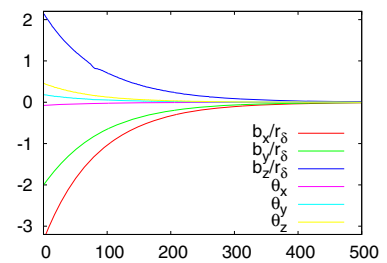

(b)

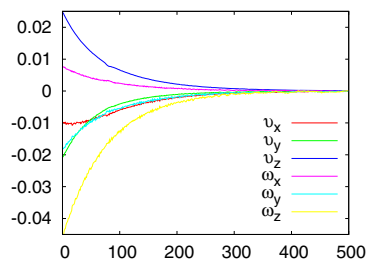

(d)
Fig. 7. Ideal case. (a) $\mathbf{s}_{\mathbf{c c}}$ error, (b) $\mathbf{s}_{\mathbf{n}}$ error. (c) and (d) Computed camera velocities $(\mathrm{m} / \mathrm{s}$ and $\mathrm{deg} / \mathrm{s})$ using $\mathbf{s}_{\mathbf{c c}}$ and $\mathbf{s}_{\mathbf{n}}$.

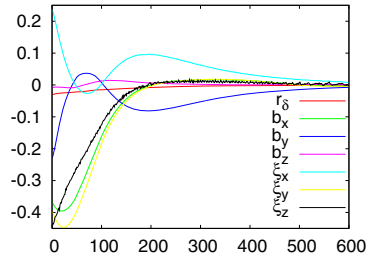

(a)

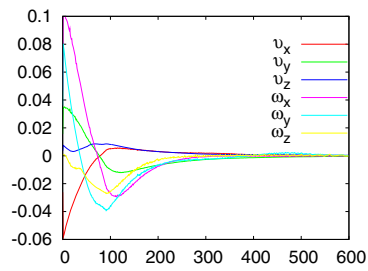

(c)

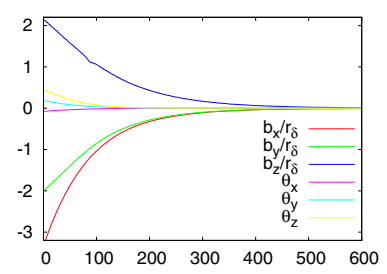

(b)

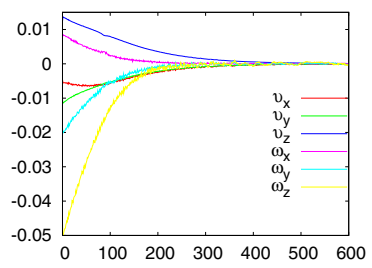

(d)
Fig. 8. Modeling error $\widehat{r}=0.5 r$. (a) s scc error, (b) $\mathbf{s}_{\mathbf{n}}$ error. (c) and (d) Computed camera velocities $(\mathrm{m} / \mathrm{s}$ and $\mathrm{deg} / \mathrm{s})$ using $\mathbf{s}_{\mathbf{c c}}$ and $\mathbf{s}_{\mathbf{n}}$.

Fig. 8 plots the results obtained in that case. It is clear from this figure that $\mathbf{s}_{\mathbf{n}}$ provides a better robustness w.r.t. modeling errors.

c) Calibration errors: Let $\left(u_{0}, v_{0}\right)$ be the pixel coordinates of the camera principal point. Let $p_{x}$ (respectively $p_{y}$ ) be the camera focal length on the $x$-axis (respectively $y$-axis). The stability to calibration errors is verified by introducing the following error in the camera intrinsic parameters: $35 \% p_{x}, 30 \% p_{y},-25 \% u_{0}$ and $27 \% v_{0}$. Once again, the system converges for both sets as shown in Fig. 9.

2) Application to a decoration balloon: We finally validate the robustness of the control law to strong modelling errors. The target is a white non-spherical decoration balloon marked with a flower picture in black. Fig. 10(b) and Fig. 10(a) picture the initial and desired images of the target respectively. Taking advantage of the stability to modeling errors, we have used an approximated radius of $6.5 \mathrm{~cm}$. In 


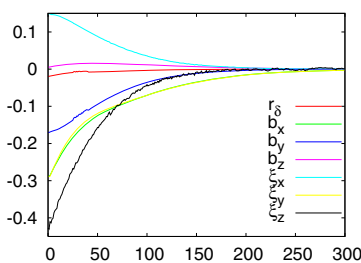

(a)

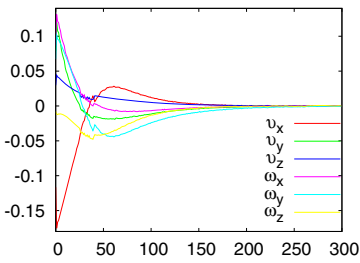

(c)

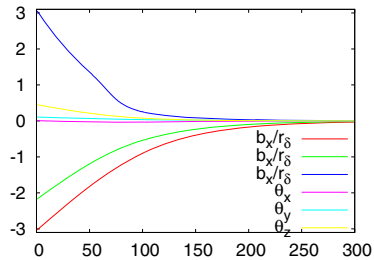

(b)

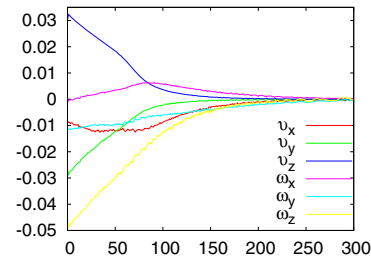

(d)
Fig. 9. Calibration errors. (a) $\mathbf{s}_{\mathbf{c c c}}$ error, (b) $\mathbf{s}_{\mathbf{n}}$ error. (c) and (d) Computed camera velocities $(\mathrm{m} / \mathrm{s}$ and $\mathrm{deg} / \mathrm{s})$ using $\mathbf{s}_{\mathbf{c c}}$ and $\mathbf{s}_{\mathbf{n}}$.

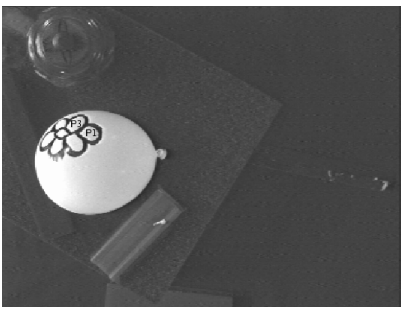

(a)

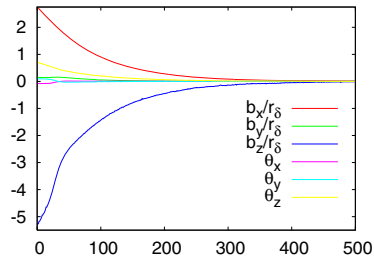

(c)

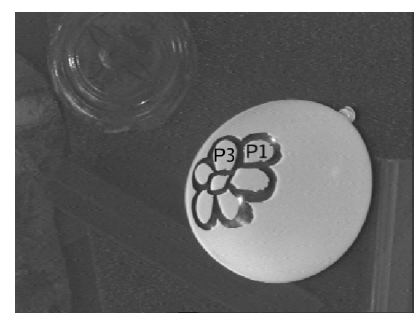

(b)

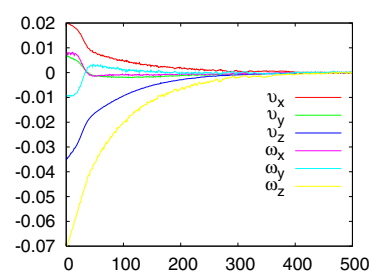

(d)
Fig. 10. New chosen set applied to a decoration balloon. (a) Desired image. (b) Initial image. (c) $\mathbf{s}_{\mathbf{n}}$ error. (d) Computed camera velocities $(\mathrm{m} / \mathrm{s}$ and $\mathrm{deg} / \mathrm{s})$.

addition, $\mathbf{P}_{1}$ and $\mathbf{P}_{3}$ have been selected as two leaves on the flower picture. Fig. 10(c) and Fig. 10(d) plot the result where we can see that the system converges. The second part of the attached video illustrates this application.

\section{CONCLUSIONS AND FUTURE WORKS}

In this paper, the spherical projection model has been exploited to design an optimal set of features for visual servoing w.r.t. two special targets: a special sphere and the CC target. For both targets, a new minimal set of six features has been selected. Regarding the stability issue, using the new set, a classical control method has been formally proved to be robust even in the presence of large modeling error.
Using a simulator, we have dismissed another set (built from the new set) as it is similar to a $3 \mathrm{D}$ visual servoing with no control of the target in the image. Using a perspective camera mounted on a six DOFs robotics system and the $\mathrm{CC}$ target, the new set has revealed a better behaviour of the system compared to the previous set recently proposed for the same target. Finally, the new set has been validated on a non-spherical decoration balloon, which shows the robustness of visual servoing w.r.t. strong modeling errors. Future works will focus on visual servoing from those two particular targets using central catadioptric cameras.

\section{ACKNOWLEDGMENTS}

The authors would like to thank N. J. Cowan for fruitful discussions; F. Janabi-Sharifi, S. Hutchinson and E. Malis for their comments on this research and earlier drafts of this paper.

\section{REFERENCES}

[1] F. Chaumette, P. Rives, and B. Espiau, "Classification and realization of the different vision-based tasks," in Visual Servoing, K. Hashimoto, Ed. Singapore: World Scientific Series in Robotics and Automated Systems, 1993, vol. 7, pp. 199-228.

[2] F. Chaumette, "Potential problems of stability and convergence in image-based and position-based visual servoing," in The Confluence of Vision and Control, D. Kriegman, G. Hager, and A. S. Morse, Eds. LNCIS Series, No 237, Springer-Verlag, 1998, pp. 66-78.

[3] W. Wilson, C. Hulls, and G. Bell, "Relative end-effector control using cartesian position-based visual servoing," IEEE Trans. on Robotics and Automation, vol. 12, no. 5, pp. 684-696, Oct. 1996.

[4] D. Dementhon and L. Davis, "Model-based object pose in 25 lines of code," Int. Journal of Computer Vision, vol. 15, no. 1/2, pp. 123-141, Jun. 1995.

[5] L. Deng, W. Wilson, and F. Janabi-Sharifi, "Dynamic perfomance of the position-based visual servoing method in the cartesian and image spaces," in IEEE/RSJ Int. Conf. on Intelligent Robots and Systems, vol. 1, Las Vegas, Nevada, Oct. 2003, pp. 510-515.

[6] P. Martinet, J. Gallice, and D. Khadraoui, "Vision based control law using 3d visual features," in World Automation Congress, WAC, Robotics and manufactoring systems, vol. 3, Montpellier, France, May 1996, pp. 497-502.

[7] E. Malis, F. Chaumette, and S. Boudet, "2 1/2 d visual servoing," IEEE Trans. on Robotics and Automation, vol. 15, no. 2, pp. 238-250, Apr. 1999.

[8] H. Hadj-Abdelkader, Y. Mezouar, and P. Martinet, "Decoupled visual servoing from set of points imaged by an omnidirectional camera," in IEEE Int. Conf. on Robotics and Automation, Rome, Italy, Apr. 2007.

[9] B. Espiau, "Effect of camera calibration errors on visual servoing in robotics," in 3rd Int. Symposium on Experimental Robotics, Kyoto, Japan, Oct. 1993.

[10] P. Corke and S. Hutchinson, "A new partitioned approach to imagebased visual visual servo control," IEEE Trans. on Robotics and Automation, vol. 17, no. 4, pp. 507-515, Aug. 2001.

[11] M. Iwatsuki and N. Okiyama, "A new formulation for visual servoing based on cylindrical coordinate system," IEEE Trans. on Robotics, vol. 21, no. 2, pp. 266-273, Apr. 2005.

[12] O. Tahri and F. Chaumette, "Point-based and region-based image moments for visual servoing of planar objects," IEEE Trans. on Robotics, vol. 21, no. 6, pp. 1116-1127, Dec. 2005.

[13] N. Cowan and D. Chang, "Geometric visual servoing," IEEE Trans. on Robotics, vol. 21, no. 6, pp. 1128-1138, Dec. 2005.

[14] D. Miller and A. Wright, "Autonomous spacecraft docking using multi-color targets," in Proceedings of the 6th Topical Meeting on Robotics, Monterey, CA, February 1995.

[15] R. Tatsambon Fomena and F. Chaumette, "Visual servoing from spheres using a spherical projection model," in IEEE Int. Conf. on Robotics and Automation, Rome, Italy, Apr. 2007. 